\title{
Ewing sarcoma in an infant and review of the literature
}

\author{
Çağlayan Selenge Bedük Esen¹, Melis Gültekin¹, G. Burça Aydın², Canan Akyüz², \\ Kader Karlı Oğuz ${ }^{3}$, Diclehan Orhan ${ }^{4}$, Mustafa Cengiz ${ }^{1}$, Murat Gürkaynak ${ }^{1}$, Ferah Yıldız ${ }^{1}$ \\ Departments of ${ }^{1}$ Radiation Oncology, ${ }^{2}$ Pediatric Oncology, ${ }^{3}$ Radiology and ${ }^{4}$ Pediatric and Perinatal Pathology, Hacettepe \\ University Faculty of Medicine, Ankara, Turkey. E-mail: melisgultekin@hacettepe.edu.tr \\ Received: 11th December 2018, Revised: 5th January 2019, Accepted: 9th January 2019
}

SUMMARY: Bedük Esen ÇS, Gültekin M, Aydın GB, Akyüz C, Karlı Oğuz K, Orhan D, Cengiz M, Gürkaynak M, Yıldız F. Ewing sarcoma in an infant and review of the literature. Turk J Pediatr 2019; 61: 760-764.

Ewing sarcoma (ES) is a rare tumor in infants and prognosis is controversial. There are no standard recommendations for treatment in such very young patients. Generally, radiotherapy (RT) is not a part of treatment in infants due to the risk of severe late side effects. In this case report, we report a 7-month-old boy with diagnosis of left mastoid bone ES with lung metastases at diagnosis, showing a rapidly fatal outcome despite aggressive systemic chemotherapy and RT without surgery.

Key words: Ewing sarcoma, infant, intracranial.

Ewing sarcoma (ES) is the second most common malignant bone tumor in children and adolescents, however, it is rarely seen in infants ( $<12$ months of age) and prognosis is controversial in this age group. ${ }^{1}$ Some studies showed inferior outcomes compared to older patients, while others showed similar results. $^{2-8}$

The standard treatment for ES includes systemic chemotherapy, surgery and/or radiotherapy (RT). Given the rarity of ES in infants, there are no standard recommendations for treatment. In such very young patients, the aim of treatment is to obtain the maximal chance of cure with minimal risk of toxicity. Generally, RT is not a part of treatment in infants due to the risk of severe late side effects. However some tumors which are not amenable for surgery may need RT as the only local control tool. In this case report, we report a 7-month-old boy with diagnosis of left mastoid bone ES with lung metastases at diagnosis showing a rapidly fatal outcome despite aggressive systemic chemotherapy and RT without surgery.

\section{Case Report}

A 7-month-old boy was referred to Hacettepe University, Department of Pediatric Oncology with sudden onset of drooping of left upper eyelid and poor feeding. The patient had no history of trauma, infection or previous medical history. He had no family history of cancer or exposure to any risk factors during pregnancy. The patient was lethargic and the physical examination revealed ptosis and restriction of movements in left eye and facial asymmetry, supporting palsies of 3 , 4 , and 6. cranial nerves and left peripheral facial paralysis and elevated intracranial pressure (ICP). A magnetic resonance imaging (MRI) scan in January 2017 showed a mass of $6.85 \times 6.1 \times 5.25 \mathrm{~cm}$ arising from the left mastoid bone. The inhomogeneous mass showed enhancement with Gadolinium-based contrast material and diffusion restriction. The left sigmoid and transvers sinuses were occluded and the brain stem and cerebellum were compressed by the tumor (Fig. 1). The patient was transferred to the intensive care unit for supportive care and elevated ICP 


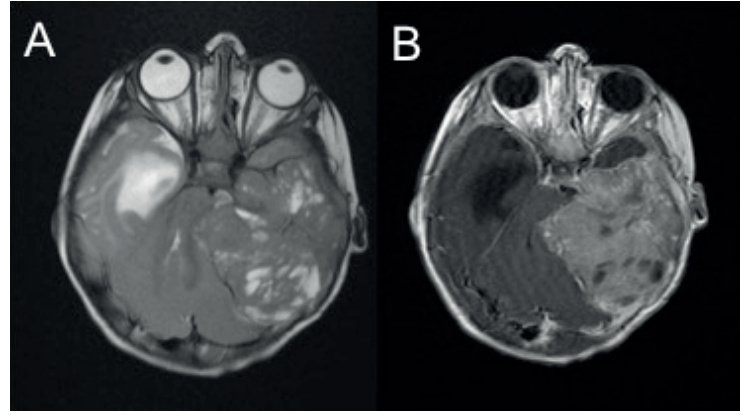

Fig. 1. Axial T2-weighted (T2W) and postgadolinium (Gd) T1W images of the cranial MRI show an inhomogeneous and enhancing mass of 6.85 x 6.1 x $5.25 \mathrm{~cm}$ size of the left mastoid bone. Please note that compression of the brainstem, cerebellum and $4^{\text {th }}$ ventricle with resultant hydrocephalus.

treatment. As soon as he was stabilized, incisional biopsy from temporal mass was performed for diagnosis. Histopathological examination revealed hypercellular small round cell tumor composed of tumor cells with scanty cytoplasm. The neoplastic cells were immunohistochemically positive for LCA, NSE, CD99, bcl-2 and vimentin, but negative for CD117, desmin, kaldesmon, S100, CD34, CD56, SMA, EMA, CD57 and myogenin (Fig. 2). Fluorescence in situ hybridization (FISH) examination revealed EWSR1 rearrangement. Pathological findings were consistent with ES/ PNET. Staging work-up was performed and computed tomography (CT) scan of the chest revealed multiple bilateral lung metastases. Chemotherapy with cisplatin was given. At the end of the first course, clinical findings of ICP resolved and he was removed to inpatient clinic. Then, European EURO-EWING regimen was planned for his tumor. The MRI after 4 cycles of chemotherapy showed prominent shrinkage of the tumor with a maximum thickness of $1 \mathrm{~cm}$ residual tumor indicating a good partial response (Fig. 3). At the end of induction courses, lung metastases disappeared, but residual tumor in temporal bone was still considered unresectable and even biopsy could not be performed although its marked regression. RT was decided to be added to treatment as local control of primary tumor. The RT was in the form of 45 Gy to initial tumor volume and followed by a 9 Gy boost to residual disease. Details of RT are summarized in Figure 4. RT was well tolerated. Only grade 1 acute dermatitis was observed on his left ear skin during RT which resolved spontaneously. Maintenance courses were given after RT. Four months after RT, a new brain MRI was performed and progression of the tumor $(2.8 \times 2.3 \times 2.8 \mathrm{~cm})$ indenting the left cerebellar hemisphere was observed (Fig. 5). Resection of the tumor was planned due to RT in-field recurrence and rapid progression of the disease (Fig. 6). However, intracranial hemorrhage leading to

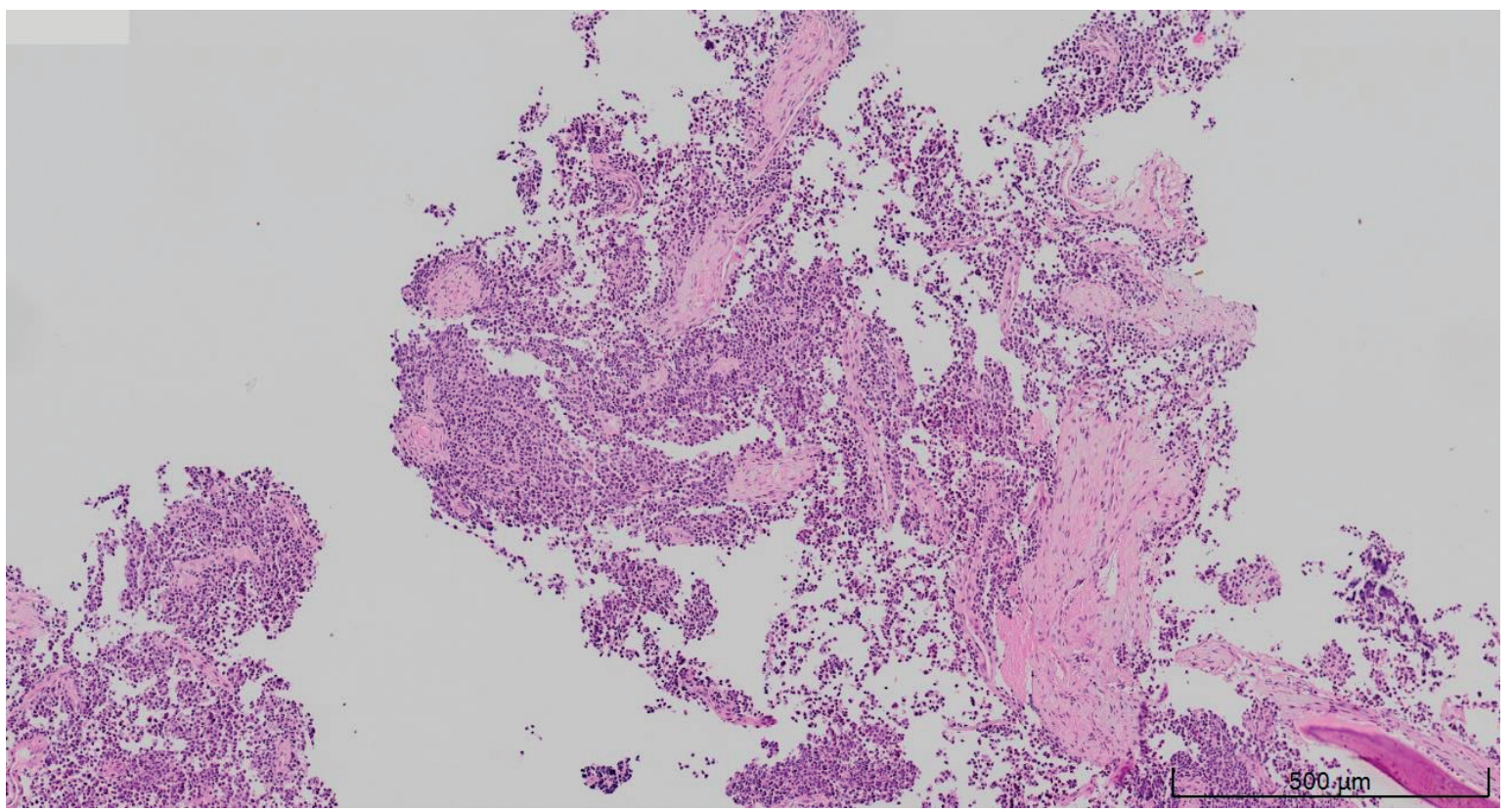

Fig. 2. Tumor composed of small round blue cells. 

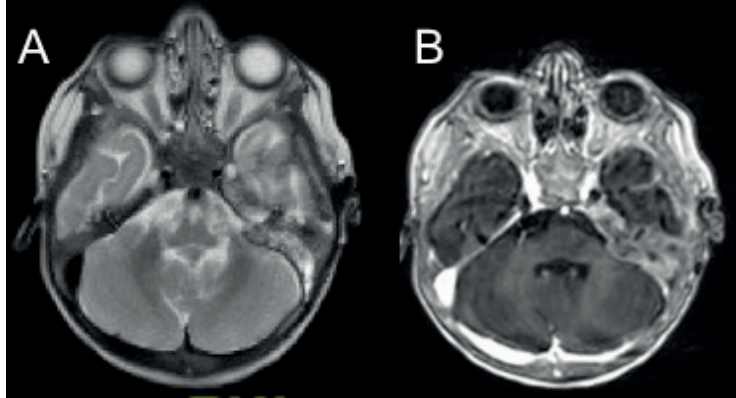

Fig. 3. Cranial MRI performed after 6 courses of chemotherapy shows marked shrinkage of the tumor with some residual of tissue of approximately $1 \mathrm{~cm}$ thickness.

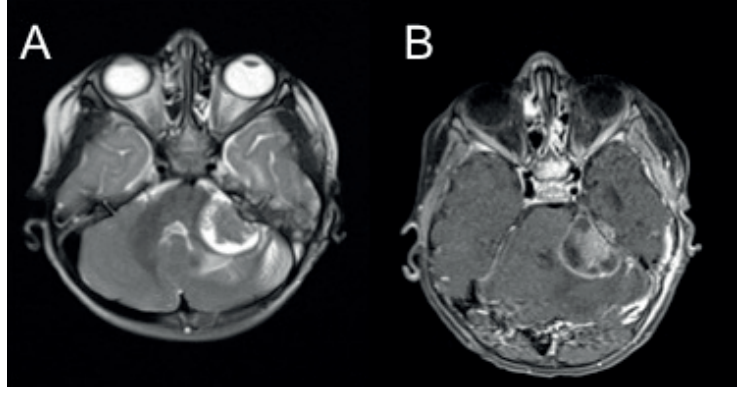

Fig. 5. Cranial MRI after RT and 3 courses of VAC chemotherapy shows a recurrent tumor involving the pontocerebellar angle.

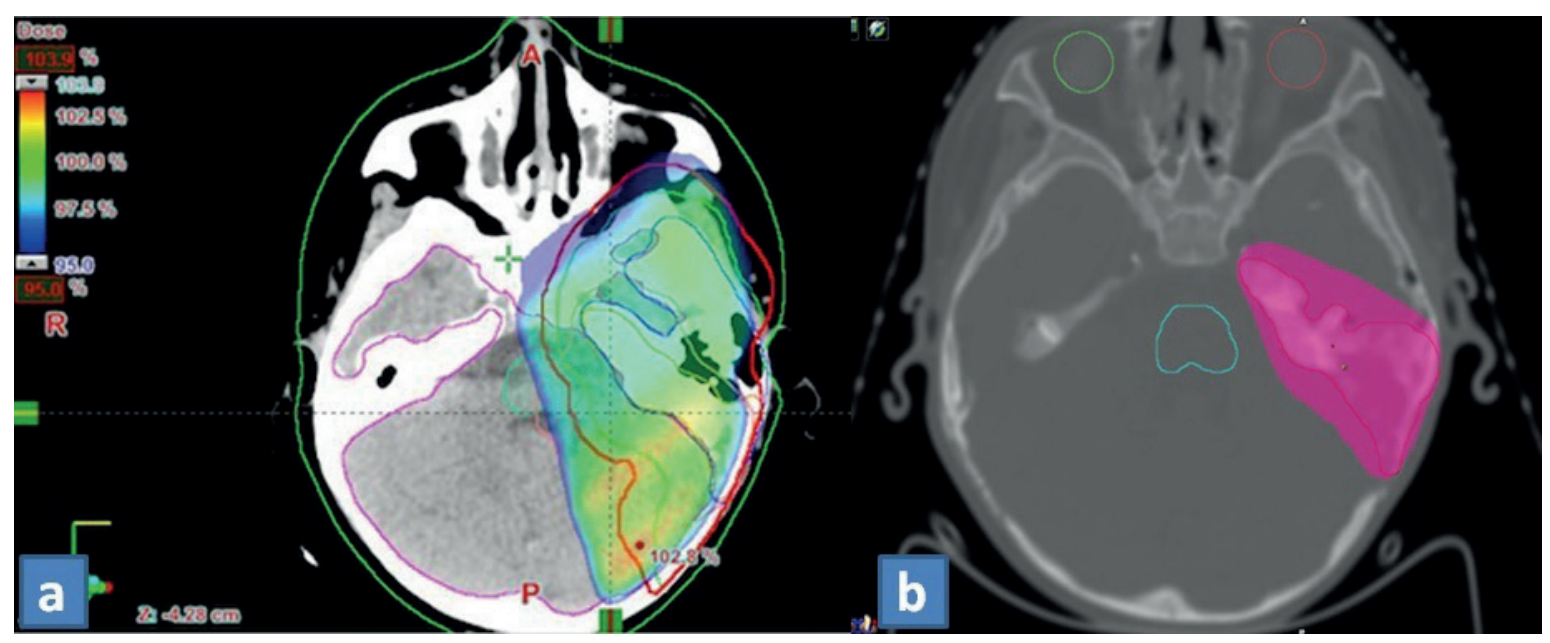

Fig. 4. Axial image of a RT planning CT and 95\% dose color wash of initial treatment volume. b. Axial image of a RT planning CT and $95 \%$ dose color wash of boost volume.

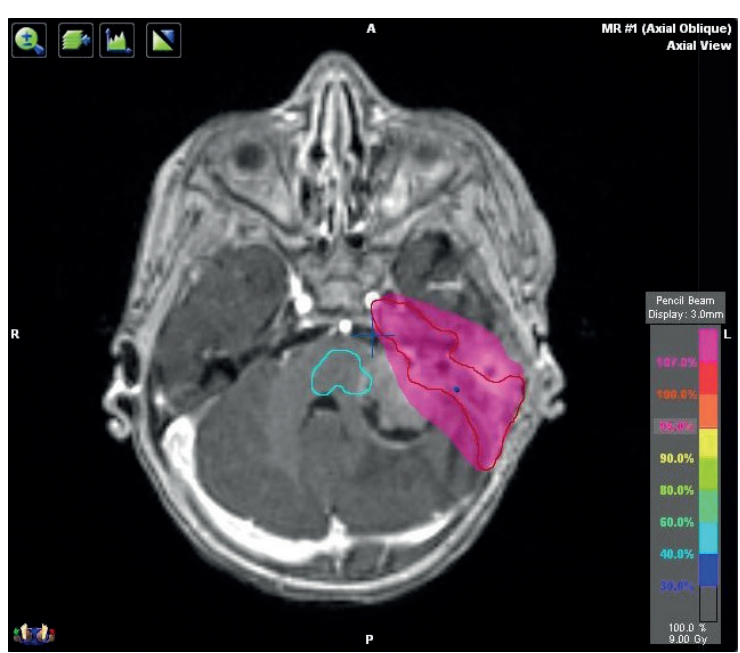

Fig. 6. Fusion image of an axial MRI and 95\% dose color wash of boost volume in case of recurrent disease. increased ICP occurred before surgery and the patient died in the intensive care unit 5 days following hemorrhage.

Written consent for publication of this case report and the accompanying images was obtained from the parents of the patient.

\section{Discussion}

ES is very rare disease in infants and it develops in various parts of the body; however it occurs most frequently in the axial skeleton., ${ }^{1,3}$ Primary intracranial localization is extremely rare, comprising approximately $1-4 \%$ of cases. ${ }^{8}$ It is most commonly originated from the dura mater with a large lesion causing neurological deficit. To the best of our knowledge, there are only four cases of ES in the literature 
with mastoid bone localization and none of these patients were infants. ${ }^{9-11}$ Recently, Yang et al. ${ }^{12}$ reported four patients with primary intracranial ES, in whom two of these were originated from temporal bone and none of them were infants. Although the infant presentation is more common in females, our case was a 7 -month-old male patient. ${ }^{4}$

Afterdiagnosticimaging studies, abiopsyshould be obtained for histopathological diagnosis to confirm ES which is characterized by small, undifferentiated blue round cell tumor with a scanty cytoplasm and hyperchromatic nucleus. Immunohistochemical studies revealed CD 99, vimentin, HBA-71, and B2 microglobulin positivity. To distinguish ES from other tumors, EWSR1 gene rearrangement should be demonstrated.

In general, clinical outcomes are controversial in infants. Kim et al. $^{6}$ reported only $5 \%$ chance of long-term survival in 21 patients with congenital ES. However, Wong et al. ${ }^{1}$ reported SEER results of 1957 patients with ES excluding intracranial localization and they found that 5-year overall survival (OS) was similar between infants and older patients. Risk of early deaths in this SEER data analysis was significantly increased in infants; however, it was unclear whether these were due to the toxicity of intensive chemotherapies or disease progression.

Currently, the standard treatment consists of systemic chemotherapy and local therapy in ES. First-line multi-agent chemotherapy includes vincristine, doxorubicin, and cyclophosphamide, alternating with ifosfamide and etoposide given for approximately 12 weeks prior to definitive local therapy and several months thereafter. ${ }^{13}$ The most important part of the treatment is local therapy which consists of maximal surgical resection or RT or a combination of both to achieve optimal local control. Whenever possible, maximal surgical resection should be performed, however, proximity of vital structures precludes complete surgery which causes poor outcomes. ES is a radiosensitive tumor so RT can be used as the sole treatment modality in tumors that cannot be resected. In the literature, patients treated with RT alone had significantly worse outcomes. ${ }^{14,15}$
Bacci et al. ${ }^{16}$ found that local failure rate was $19 \%, 9 \%$, and $11 \%$ in patients treated with RT alone, surgery alone, and surgery and RT, respectively. However, many irradiated patients had inoperable tumors in unfavorable locations (pelvis, spine, intracranial etc.) that may have biased the results.

The optimal therapy for infants and other young patients with ES is not known due to its rarity. ${ }^{1}$ These patients are often treated similarly to older children and adults. The aim of treatment is to obtain the maximal chance of cure with minimal risk of toxicity. However, RT is not often utilized to infants due to the risk of long-term toxicity. ${ }^{1,17}$ Similarly, most of the infants cannot tolerate intensive chemotherapy. ${ }^{18}$ Although the Euro-Ewing 1999 protocol recommends reducing the chemotherapy dose in these patients, there are no special recommendations for RT field or dose dedicated to infants. However, in many types of tumors (e.g. medulloblastoma), it is recommended to reduce RT dose for patients less than 3 years of age. Although there is no clear dose-response relationship in ES, $\geq 54 \mathrm{~Gy}$ is recommended for definitive cases. ${ }^{19-21}$ In our patient, Euro-Ewing chemotherapy protocol was administered and he was treated with RT as a sole local treatment. Despite high dose $\mathrm{RT}$, the patient developed disease progression which was detected four months after RT.

About $25 \%$ of patients with ES presents with metastatic disease at diagnosis. ${ }^{22}$ The most common site of metastasis is the lung, followed by bone. Though ES of the intracranial region has been less frequently reported to present with metastases at diagnosis, our case was with multiple lung metastases at diagnosis. Current treatment protocols also include curative treatment approach for patients with metastatic disease. Whole lung RT following a complete response to conventional chemotherapy is a standard treatment for patients with metastatic disease limited to the lungs. ${ }^{23}$ Due to his very young age, our patient was not treated with whole lung RT and close follow-up was preferred.

In conclusion, ES in infants is a rare disease and the optimal treatment approach and the prognostic significance of age is not well defined. To reduce the intensification of 
treatment protocol both in chemotherapy and RT and/or surgery may lead to worse outcomes in these patients. Intensive chemotherapy and full dose RT should be used in these patients when complete resection could not be achieved.

\section{REFERENCES}

1. Wong T, Goldsby RE, Wustrack R, Cash T, Isakoff MS, DuBois SG. Clinical features and outcomes of infants with Ewing sarcoma under 12 months of age. Pediatr Blood Cancer 2015; 62: 1947-1951.

2. Rodriguez-Galindo C, Liu T, Krasin MJ, et al. Analysis of prognostic factors in ewing sarcoma family of tumors: review of St. Jude Children's Research Hospital studies. Cancer 2007; 110: 375-384.

3. van den Berg H, Dirksen U, Ranft A, Jurgens H. Ewing tumors in infants. Pediatr Blood Cancer 2008; 50: 761-764.

4. Maygarden SJ, Askin FB, Siegal GP, et al. Ewing sarcoma of bone in infants and toddlers. A clinicopathologic report from the Intergroup Ewing's Study. Cancer 1993; 71: 2109-2118.

5. Duchman KR, Gao Y, Miller BJ. Prognostic factors for survival in patients with Ewing's sarcoma using the surveillance, epidemiology, and end results (SEER) program database. Cancer Epidemiol 2015; 39: 189195.

6. Kim SY, Tsokos M, Helman LJ. Dilemmas associated with congenital ewing sarcoma family tumors. J Pediatr Hematol Oncol 2008; 30: 4-7.

7. Akcali M, Yapicioglu H, Akay E, et al. A congenital soft tissue Ewing sarcoma in a newborn patient. Turk J Pediatr 2017; 59: 76-79.

8. Salunke PS, Gupta K, Malik V, et al. Primary Ewing's sarcoma of cranial bones: analysis of ten patients. Acta Neurochir (Wien) 2011; 153: 1477-1485.

9. Egan G, Pierro J, Madhusoodhan PP, Ilyas G, Cohen B, Bhatla T. Primary Ewing sarcoma of the mastoid: A novel case mimicking cute mastoiditis. J Pediatr Hematol Oncol 2018; 40: 148-151.

10. Garcia-Perez EA, Nunez-Ferrer P. Primary intracranial Ewing's sarcoma of the mastoid bone. A case report. Rev Neurol 2003; 36: 340-342.

11. Zimmermann JL. Ewing Sarcoma of the mastoid. Penn Med J 1934; 37: 654-656.

12. Yang MJ, Whelan $\mathrm{R}$, Madden $\mathrm{J}$, et al. Intracranial Ewing sarcoma: four pediatric examples. Childs Nerv Syst 2018; 34: 441-448.
13. Choudhury KB, Sharma S, Kothari R, Majumder A Primary extraosseous intracranial Ewing's sarcoma: Case report and literature review. Indian J Med Paediatr Oncol 2011; 32: 118-121.

14. Ahmed SK, Randall RL, DuBois SG, et al. Identification of patients with localized Ewing sarcoma at higher risk for local failure: A report from the Children's Oncology Group. Int J Radiat Oncol Biol Phys 2017; 99: 1286-1294.

15. Becker RG, Gregianin LJ, Galia CR, et al; Brazilian Collaborative Study Group of Ewing Family of Tumors - EWING1 and the Brazilian Society of Pediatric Oncology - SOBOPE. What is the impact of local control in Ewing sarcoma: analysis of the first Brazilian collaborative study group - EWING1. BMC Cancer 2017; 17: 420.

16. Bacci G, Longhi A, Briccoli A, Bertoni F, Versari M, Picci $P$. The role of surgical margins in treatment of Ewing's sarcoma family tumors: experience of a single institution with 512 patients treated with adjuvant and neoadjuvant chemotherapy. Int J Radiat Oncol Biol Phys 2006; 65: 766-772.

17. Carlotti CGJ, Drake JM, Hladky JP, Teshima I, Becker LE, Rutka JT. Primary Ewing's sarcoma of the skull in children. Utility of molecular diagnostics, surgery and adjuvant therapies. Pediatr Neurosurg 1999; 31: 307-315.

18. Sharib JM, Cyrus J, Horvai A, et al. Predictors of acute chemotherapy-associated toxicity in patients with Ewing sarcoma. Pediatr Blood Cancer 2012; 59: 611-616

19. Nesbit ME Jr, Gehan EA, Burgert EO Jr, et al Multimodal therapy for the management of primary, nonmetastatic Ewing's sarcoma of bone: a long-term follow-up of the First Intergroup study. J Clin Oncol 1990; 8: 1664-1674.

20. Paulino AC, Nguyen TX, Mai WY, Teh BS, Wen BC. Dose response and local control using radiotherapy in non-metastatic Ewing sarcoma. Pediatr Blood Cancer 2007; 49: 145-148.

21. Talleur AC, Navid F, Spunt SL, et al. Limited margin radiotherapy for children and young adults with Ewing sarcoma achieves high rates of local tumor control. Int J Radiat Oncol Biol Phys 2016; 96: 119126.

22. Esiashvili N, Goodman M, Marcus RBJr. Changes in incidence and survival of Ewing sarcoma patients over the past 3 decades: Surveillance Epidemiology and End Results data. J Pediatr Hematol Oncol 2008; 30: $425-430$

23. Paulussen $M$, Ahrens S, Burdach S, et al. Primary metastatic (stage IV) Ewing tumor: survival analysis of 171 patients from the EICESS studies. European Intergroup Cooperative Ewing Sarcoma Studies. Ann Oncol 1998; 9: 275-281. 